\title{
An Optical P - L Relation for LPVs
}

\author{
M.J. Pierce ${ }^{1}$, D.R. Crabtree ${ }^{2}$, \\ ${ }^{1}$ Kitt Peak National Obs., U.S.A., ${ }^{2}$ Dominion Astrophysical Obs., Canada
}

\section{An Optical P-L Relation for LPVs}

Supergiant Long-Period Variables (LPVs) are both more luminous and more common than Cepheids. The fact that a P - L relation exists at K (e.g. Wood et al. $1983 \mathrm{ApJ}$, 272, 99; Mould et al. 1990 ApJ, 349, 503) and not at visible wavelengths is usually attributed to the strong temperature sensitivity of TiO, which dominates the spectra of these stars in the visible. This has led us to apply a narrow bandpass $\left(\lambda_{0}=8250 \AA\right.$, $B W=350 \AA$ ), avoiding $\mathrm{TiO}$, in the hopes that an optical $\mathrm{P}-\mathrm{L}$ relation can be found.

\section{Estimated Distances for the LMC and M 33}

The data for Per OB1, the LMC, and M 33 are shown in figure 1. The small dispersion $\left(\sigma_{L M C}=0.22\right.$ mag. $)$ and flat slope are evident. Combining the data we find:

$$
M_{I}=-0.00267 P(\text { days })-6.31 \text {, }
$$

with the absolute calibration from the ten LPVs in Per OB1, assuming $m-M=$ $11.8 \pm 0.2$ (Garmany \& Stencel $1992 \mathrm{AAS}, \mathbf{9 4}, 211$ ). This results in estimated distance moduli of $18.33 \pm 0.05$, and $24.79 \pm 0.08$ for the $L M C$ and $M 33$, vs. $18.47 \pm 0.15$, and $26.64 \pm 0.09$ using Cepheids (e.g. Feast \& Walker 1987 ARAA, 25, 345; Freedman et al. 1991 ApJ, 372, 455). Evidently, any systematics from metallicity differences must be small. We conclude that this $\mathrm{P}-\mathrm{L}$ relation for LPVs is a powerful tool for estimating extragalactic distances and offers a useful compliment/alternative to Cepheids. LPVs are being detected in Virgo Cluster spirals (Pierce et al. this volume).

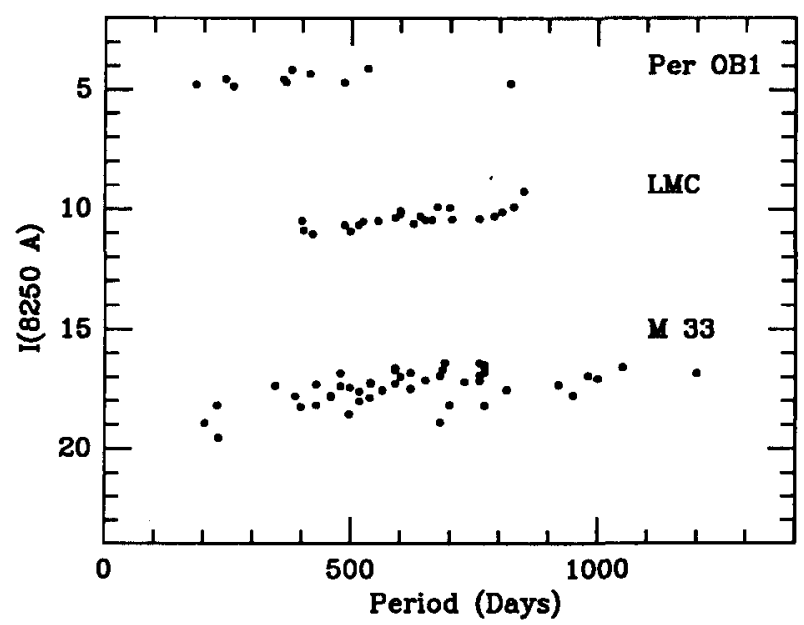

102 